\title{
A EUROPEAN BENCHMARKING OF CONTINUITY OF SUPPLY REGULATION
}

\author{
Werner FRIEDL \\ E-Control - Austria \\ werner.friedl@e-control.at \\ Riccardo VAILATI \\ (formerly with AEEG) - Italy \\ unitaQSM@autorita.energia.it \\ Math BOLLEN \\ LTU - Sweden \\ math.bollen@ltu.se
}

\author{
Roland GÖRLICH \\ E-Control - Austria \\ roland.goerlich@e-control.at \\ Romain CASTEL \\ CRE - France \\ romain.castel@cre.fr \\ David Batic \\ AGEN-RS - Slovenia \\ david.batic@agen-rs.si
}

\author{
Ognjen RADOVIC \\ E-Control - Austria \\ ognjen.radovic@e-control.at \\ Antonio CANDELA \\ $\mathrm{CNE}$ - Spain \\ acm@cne.es
}

\begin{abstract}
The Council of European Energy Regulators has been publishing Benchmarking Reports on the Quality of Electricity Supply since 2001. For the $5^{\text {th }}$ edition of the Benchmarking Report the 29 member countries of CEER were joined by the 9 NRAs from the Energy Community and the NRA from Switzerland. This paper contains the main results, findings and recommendations on continuity of supply from the latest edition of the Benchmarking Report.
\end{abstract}

\section{INTRODUCTION}

The Council of European Energy Regulators (CEER) [1] periodically surveys and analyses the quality of electricity supply in its member countries (27 member states of the European Union, Iceland and Norway), addressing three major aspects: the availability of electricity (continuity of supply), its technical properties (voltage quality) and the speed and accuracy with which customer requests are handled (commercial quality).

These surveys and analyses take the form of CEER Benchmarking Reports on Quality of Electricity Supply. The first report was issued in 2001 [2], followed by the second, third and fourth editions in 2003, 2005 and 2008 respectively [3] [4] [5]. Similarly, information on the national regulations and its effects in the Energy Community were gathered by the Energy Community Regulatory Board (ECRB) in the 2009 ECRB Report on the Quality of Electricity Service Standards and Incentives in Quality Regulation [6].

In addition to National Regulatory Authorities (NRAs) from CEER member countries, the 9 NRAs from the Energy Community [7] and the National Regulatory Authority of Switzerland joined for the 2011 Benchmarking Report (BR) [8].

A detailed survey was sent out in March 2011 to NRAs in the CEER member countries and in the Energy Community Contracting Parties (CPs) to obtain information on all three aspects of quality of supply. The survey contained detailed questions about existing and planned regulations on quality of supply, monitoring practices as well as questions on existing quality levels.

In this paper, we present the main results from the 2011 edition as well as the recommendations from CEER and ECRB on continuity of supply $(\mathrm{CoS})$. The results on voltage quality and commercial quality are presented in [9] and [10].

\section{CONTINUITY OF SUPPLY MONITORING}

All countries who participated on this survey (27 CEER members/observers and 9 NRAs from ECRB) stated that $\mathrm{CoS}$ is monitored within their electricity networks countrywide. This monitoring is done in different ways in different countries. Differences between countries include the kind of interruptions monitored; the level of detail being reported; the interpretation and highlighting of various indicators; but also definitions used.

Differences in definitions for long, short and transient interruptions (concerning mainly the specifications for duration of an interruption) are reported for different countries. 27 countries define short interruptions. Among these countries, 14 record these interruptions separately. Meanwhile, 3 countries monitor interruptions shorter than three minutes without distinction and separate definition. 4 countries record transient interruptions (the ones with the shortest duration) separately. Some countries monitor transient interruptions together with short interruptions.

Most countries use separate classifications for planned and unplanned interruptions. In most countries advance notification is sufficient for an interruption to be classified as a planned interruption. 34 out of 36 countries monitor planned and unplanned interruptions separately. Whereas there is general agreement on the definition of a planned interruption, the requirement for advance notice varies strongly between countries (between 24 hours and 50 days).

Not all countries monitor interruptions at all voltage levels, but all of them generate statistic records for incidents at more than one voltage level. Medium voltage (MV) and high voltage (HV) levels are monitored in all countries. 
Incidents in the transmission network are monitored in 25 of the 36 countries. Incidents at all voltage levels are monitored in 18 countries.

Nearly half of the countries use automatic logging, automatic identifications, or both when measuring long and short interruptions. 31 out of 36 countries use indices to monitor both frequency and duration of long interruptions, for both planned and unplanned interruptions. The outcome of the survey shows clearly that a range of indicators is in use in different countries. The use of multiple indicators to quantify the $\mathrm{CoS}$ results in more information being available and more possibilities to observe trends.

SAIDI and SAIFI are the basic indices, reported in almost all countries, albeit under different names and with different methods for weighting the interruptions.

\section{ANALYSIS OF NATIONAL DATA}

As mentioned before, European countries use different indicators and different weighting methods when evaluating interruptions. Two main groups of indicators - "minutes lost per year" (SAIDI) and "number of interruptions per year" (SAIFI) - are collected by countries. In addition to the monitoring of duration and frequency of interruptions, one can also examine whether the interruptions were planned or unplanned. The occurrences which are considered an exceptional event can be determined in different ways. Some countries have a statistical approach and others focus their definition on the causes of exceptional events.

When interpreting the results and especially when comparing between countries, one should consider the differences in calculation of the indices, in definitions of exceptional event and in the voltage levels at which incidents are monitored. Despite the difference in names and calculation methods between countries, the results can be shown in the same diagram.

For both groups of indicators the curves per country of unplanned long interruptions (excluding exceptional events) show a smooth trend change (see also Fig. 1 for CEER countries), generally decreasing or being constant. Especially from 2004 onwards, the decreasing trend in the total amount of minutes lost (i.e. improving $\mathrm{CoS}$ ) is reaching a stable level, with some countries having about 30 minutes lost per year and the majority of countries below 100 minutes per year. That being said, increases in the total number of minutes lost have been observed in a few countries. Considering the data for the period since the last $\mathrm{BR}$, same quality levels or a smooth general tendency for increase in quality can be observed in nearly all countries.

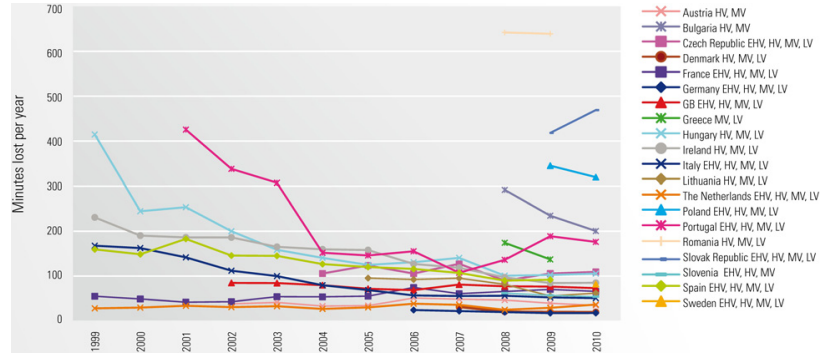

Fig. 1. Unplanned long interruptions excluding exceptional events; minutes lost per year

The CoS indicators of unplanned long interruptions including all events (without removing exceptional events from the statistics) show much larger year-to-year variations than the filtered values in Fig. 1 whereby the year-to-year variation in the number of interruptions is less than the variation for minutes lost: extreme events result in longer interruptions more often than in more interruptions.

The values for minutes lost per year due to planned interruptions (not shown in this paper) show a very wide spread between the countries, from less than 10 minutes per year to over 400 minutes per year. No trends are visible in the figures; the minutes lost due to planned interruptions remain more or less constant during the observation period, although some countries show a minor reduction. The differences between states may be due to the way in which the distribution network is designed (with or without redundant supply paths) and the amount of maintenance and other works in the distribution network. A temporary high level of planned interruptions could be a sign of investments in the distribution networks, aiming at reducing the number of unplanned interruptions in the future. High levels of planned interruptions can also be due to replacement and repair of components that were provisionally restored after a major storm and due to a widespread replacement of energy meters.

\section{ANALYSIS OF DISAGGREGATED DATA}

The analysis of interruptions in rural and urban networks (available data from 5 countries) shows that definitions of different types of areas differ significantly but it can also be shown that $\mathrm{CoS}$ improves when moving from rural to suburban to urban areas. The values for the minutes lost during this kind of interruption for the three areas are similar in almost all countries and are decreasing constantly.

Although few countries have provided reliable SAIDI data according to the voltage level of the incidents, the data still clearly indicates that around 70\%/85\% (for CEER/ECRB) of both SAIDI and SAIFI for LV users are caused by incidents on MV networks.

Another aim of the analysis was to establish whether a correlation exists at European level between the $\mathrm{CoS}$ and the technical characteristics of the network. The analysis focuses in particular on the percentage of underground 
cables in distribution networks, as this is often considered to have a significant impact on the $\mathrm{CoS}$ and is easy to quantify.

Linear regressions are used to evaluate the correlation between the percentage of underground cables in the distribution networks and the CoS, country per country. Even if datasets are too small to give robust results (analysis are based on replies from 18 countries), a clear pattern emerges, as expected: the higher the rate of undergrounding, the better the CoS. This pattern is consistent with the usual statements on the issue:

- underground cables are protected from several very common causes of incident, and therefore have a lower failure rate (number of failures per year) than overhead lines;

- in particular, they are far less prone to widespread failures, mostly caused by storms, than overhead lines;

- they do have several downsides: they are more difficult to repair, sometimes damaged by earthworks and more affected by some specific natural events (for example floods and earthquakes), even though these events are generally rare;

- the downsides are not sufficient to offset the benefits, and $\mathrm{CoS}$ benefits from undergrounding.

Yet this result should be interpreted with care. Indeed, CoS depends on a variety of parameters that can vary widely from country to country (e.g. population density, countries topology, climate, history behind the construction and the evolution of the electricity network), which makes it difficult to analyse the specific impact of the percentage of undergrounding on the $\mathrm{CoS}$ independently from the other parameters. It is also important to note that a strong statistical correlation between two indicators does not imply that one is the main cause of the other. In the present case, the many parameters that impact the $\operatorname{CoS}$ are certainly correlated to a certain extent. Additionally, the analysis does not include a cost-benefit analysis of the impact of the percentage of underground cables on the level of CoS. Incidentally, if it is generally accepted that undergrounding the networks improves $\mathrm{CoS}$, it is also often accepted that, for the sole purpose of improving $\mathrm{CoS}$, its cost-benefit balance is in general rather low compared to some other possible solutions. Therefore, no conclusion can be drawn regarding the cost-benefit balance of undergrounding the networks, for the sole purpose of improving CoS, apart from recommending cost-benefit analysis to improve the efficiency of expenditures.

\section{CONTINUITY OF SUPPLY REGULATION}

The CEER survey provides an overview of the existing quality regulation frameworks in CEER countries (for distribution and for transmission networks) and places a special focus on general experiences and those implementation processes as well as possible future improvements of the systems in place.
The measurement of actual continuity levels through indicators and standards constitutes the basis for regulating continuity and quality of supply as a whole. In general, the actual measurement of continuity can be performed on two different levels, namely system level and user-specific level. While the measurement at system level is usually done on an aggregate basis, measurement at user level is usually based on surveys asking customers about their satisfaction, expectations, willingness to pay for high quality or willingness to accept lower quality levels.

TABLE I

CONTINUITY OF SUPPLY REGULATION AT SYSTEM-LEVEL

\begin{tabular}{|l|c|c|c|}
\hline & Rewards & Penalties & Combination \\
\hline Distribution & - & DK, HU, IT & $\begin{array}{c}\text { BG, FI, FR, GB, } \\
\text { IE, IT, LT, NL, } \\
\text { NO, PT, SI, SE, ES }\end{array}$ \\
\hline Transmission & ES & DK, HU, IT & $\begin{array}{c}\text { FI, FR, GB, IE, IT, } \\
\text { LT, NO, PT }\end{array}$ \\
\hline $\begin{array}{l}\text { No existing } \\
\text { CoS scheme }\end{array}$ & \multicolumn{2}{|c|}{ AT, CY, CZ, EE, DE, GR, LV, LU, PL, RO, SK } \\
\hline
\end{tabular}

Intention/plans for implementation of a $\mathrm{CoS}$ regulation at system level has: AT, CZ, DE, GR, LU and RO.

Various countries employ incentives at single-user level; 18 CEER countries offer individual compensation to network users when standards are not met. Individual compensation is actually not in place in 8 countries. However, some countries are planning to introduce compensation payments in the future. In 16 countries, the network user has the right to be reimbursed (or to receive reduction of network tariffs) after a very long interruption. In 4 countries, compensation relates to a maximum number of interruptions in one year. In 5 countries, compensation applies for planned interruptions, with different implementation solutions (related to the duration or to the notice). In some countries, customer research has been used to determine the compensation level for interruptions at the individual customer level.

Other countries have different methods to determine compensation, such as estimation of the cost of the interruption, percentage of yearly network tariff or international comparison.

Pursuing an optimal level of CoS, improving the performance of network operators, sustaining a high level of electricity quality and eliminating differences between the $\mathrm{CoS}$ in different distribution areas were just some of the reasons cited for introducing incentive regimes. Implementation did not commence simultaneously in every location. Moreover, the monetary effects of regulation were sometimes delayed with respect to the start of the regulation The incentive regimes have already been changed in certain countries and without a doubt, quality incentive regulation will change in the future. Many countries that have not yet implemented it will do so, while others will focus on improving their regulation.

The development of regulation frameworks in the CPs of the Energy Community is on an initial stage in the 
prevailing number of cases. The main emphasis in the CPs is put on CoS monitoring, but one country (Moldova) already developed individual (customer based) and system standards and, accordingly, a compensation scheme and reward/penalty scheme are applied. However, it can be concluded that activities for implementing continuity standards and incentive schemes are ongoing or will start soon - it can be expected that other CPs will follow and develop their frameworks till 2015.

\section{CONCLUSIONS}

A number of findings and recommendations were obtained from the survey results, the analysis of the survey results, and the subsequent discussions within CEER and the Energy Community. For specific details and exact formulations, the reader is referred to the text of the $5^{\text {th }}$ edition of the BR [8]. Strongly summarized formulations of those findings and recommendations are:

$\checkmark$ Finding \#1: Continuity of supply is monitored in all countries

$\checkmark$ Recommendation \#1: Expand the monitoring of continuity of supply at all voltage levels and all interruption durations

$\checkmark$ Finding \#2: Continuity of supply indicators, procedures for data collection vary across countries

$\checkmark$ Recommendation \#2: Harmonise continuity of supply indicators and data collection procedures

$\checkmark$ Finding \#3: Continuity of supply improvements tend to become stable

$\checkmark$ Recommendation \#3: Investigate continuity of supply trends for a periodic review of regulation.

$\checkmark$ Finding \#4: Continuity of supply varies depending on the population density and the voltage level

$\checkmark$ Recommendation \#4: Assess disaggregated continuity data in order to identify priorities

$\checkmark$ Finding \#5: Continuity of supply levels are affected by network characteristics

$\checkmark$ Recommendation \#5: Promote cost-benefit analysis to improve the efficiency of expenditure on networks

$\checkmark$ Finding \#6: Incentive schemes are used to regulate continuity of supply in distribution and transmission networks

$\checkmark$ Recommendation \#6: Implement an incentive scheme for maintaining or improving general continuity levels

$\checkmark$ Finding \#7: Incentive schemes for individual continuity levels are used in many countries and have different formulations

$\checkmark$ Recommendation \#7: Implement compensation payments for network users affected by very long interruptions

$\checkmark$ Finding \#8: More countries participate in benchmarking continuity

$\checkmark$ Recommendation \#8: Exchange information on continuity of supply and its regulation

All finding and recommendations produced by CEER
NRAs are relevant to Energy Community Contracting Partners but with somewhat different significance and urgency. This may be perceived through findings and recommendations of ECRB presented in the Annex to [8]. The BRs have demonstrated the importance of a continued exchange of information on quality indicators, actual quality levels, standards, regulatory mechanisms and strategies. Their publication has facilitated obtaining information on the regulation of quality and on the effects of this regulation in different European countries. Good practices for monitoring and regulating quality in electrical networks are described in the $5^{\text {th }}$ edition of the BR and summarized in this paper. The findings and recommendations will form a basis for further development of regulation and monitoring. It is important that NRAs continue exchanging best practices for regulating electrical network industries, as done in the BRs.

\section{ACKNOWLEDGEMENT}

The contributions are acknowledged from many others to the material that formed the basis for this paper, especially the other members of the Electricity Quality of Supply and Smart Grids Task Force within CEER and the ECRB Customer Working Group within the Energy Community. The text of this paper is based on the text in the $5^{\text {th }}$ Benchmarking Report on Quality of Electricity Supply. Where the text of this paper deviates from the text of the Benchmarking Report, it is the personal opinion of the authors and not necessarily the opinion of CEER or ECRB, neither of the national regulatory authorities involved.

\section{REFERENCES}

[1] Council of European Energy Regulators, http://www.energy-regulators.eu

[2] Quality of Electricity Supply: Initial Benchmarking on Actual Levels, Standards and Regulatory Strategies", CEER, April 2001.

[3] Second Benchmarking Report on Quality of Electricity Supply, CEER, September 2003.

[4] Third Benchmarking Report on Quality of Electricity Supply 2005, CEER, December 2005.

[5] $4^{\text {th }}$ Benchmarking Report on Quality of Electricity Supply, CEER, December 2008.

[6] ECRB Report on the Quality of Electricity Service Standards and Incentives in Quality Regulation, ECRB, July 2009.

[7] Energy Community, http://www.energycommunity.org

[8] $5^{\text {th }}$ Benchmarking Report on Quality of Electricity Supply, CEER and ECRB, December 2011.

[9] M. Bollen et al., A European benchmarking of voltage quality regulation. This conference.

[10] A. Mozsolics, et al., A European benchmarking on commercial quality regulation. This conference. 